\title{
Introduction to the brain imaging and behavior special issue: mild traumatic brain injury among active duty service members and veterans
}

\author{
David F. Tate ${ }^{1}$ - Elisabeth A. Wilde $2,3,4$. Sylvain Bouix ${ }^{5} \cdot$ Stephen R. McCauley ${ }^{2,3,4}$
}

Published online: 30 August 2015

(C) Springer Science+Business Media New York 2015

As noted in the recent special issue in Brain Imaging and Behavior (Tate et al. 2012), there has been a growing interest among researchers, clinicians, policy makers, patients and family members, and other consumers regarding mild traumatic brain injury (mTBI). This interest stems from several converging points of view including epidemiology data that indicates the significant prevalence and incidence of mTBI in the general population, associated legal issues (i.e., symptom validity), our growing understanding of the effects of concussive and subconcussive sports injuries (i.e., chronic traumatic encephalopathy), and an attentiveness to the relationship between TBI and dementia in a rapidly aging society. These interests combined with recent advances in cognitive sciences, improvement in the clinical management of mTBI, a growing number of treatment options, and acquisition and analysis of imaging data are propelling the effort

David F. Tate

David.Tate@mimh.edu

Elisabeth A. Wilde

ewilde@bcm.edu

1 Missouri Institute of Mental Health, University of Missouri-St. Louis, 4633 World Parkway Circle, Berkeley, MO 63134-3115, USA

2 Department of Physical Medicine and Rehabilitation, Michael E. DeBakey VA Medical Center and Baylor College of Medicine, BCM637, Houston, TX, USA

3 Department of Neurology, Michael E. DeBakey VA Medical Center and Baylor College of Medicine, BCM637, Houston, TX, USA

4 Department of Radiology, Michael E. DeBakey VA Medical Center and Baylor College of Medicine, BCM637, Houston, TX, USA

5 Brigham and Women's Hospital, Harvard Medical School, Boston, MA, USA toward identification of unique biomarkers that could improve diagnosis, prognosis, and guide/assess treatments.

Not surprisingly, mTBI occurs in the context of a number of different situations. However, in addition to common means of head injury among civilians (i.e., motor vehicle accidents, falls, and other collision-related events), Military and Veteran populations have a number of additional deployment-related risk factors for head injury including combatant training, gunshot wounds, blast exposure, and assault. Brain-related injury in many Military and Veteran TBI patients is also complicated by additional comorbid conditions not typically experienced by civilian TBI patients including combat-related PTSD or depression, burns, hearing dysfunction, traumatic amputations and other injuries associated with combat.

With the recent combat operations in support of Operation Enduring Freedom (OEF), Operation Iraqi Freedom (OIF), Operation New Dawn (OND), and other conflicts, surveillance data from the Defense and Veterans Brain Injury Centers (DVBIC) have identified over 327,299 U.S. Service Members and Veterans across the branches of service that sustained a TBI (https://dvbic.dcoe.mil/sites/default/files/DoD-TBI-WorldwideTotals\%202000-2015Q1-May15-2015.pdf). These head injuries occur both during theatre operations and in garrison with the majority $(>70 \%)$ falling into the mild range of severity. The sheer numbers of Service Members and Veterans injured underscore the importance of advancing our understanding of the functional, clinical, and long-term consequences of mild TBI, especially in Military populations. Moreover, there is a growing interest in understanding the impact of mTBI on unit readiness, return to duty, safety of troop operations, troop retention, and long-term cognitive effects (i.e., chronic traumatic encephalopathy and possible links with dementia). Given these concerns, there has been a sudden and rapid growth in the number of investigators 
examining clinical and cognitive effects of mTBI in Military and Veteran populations, and together these studies have led to advancement in several areas including the development of potential diagnostic biomarkers.

Given the unique clinical and sociological features of these patients, we have endeavored to assemble a special issue with an emphasis on mTBI. This brings together a number of review and original articles highlighting and addressing unique clinical features of this population. We begin this special issue with several high level reviews covering important topic content areas including military epidemiology, medical imaging, traditional/non-traditional therapeutic interventions, and symptom validity that provide a broad basis for understanding the current state of the literature as well as identifying a number of future directions. Helmick and colleagues review the epidemiology and treatment of mild TBI in Military and Veteran populations. They describe current standards and clinical practice guidelines for identifying, categorizing, and treating TBI in the military, arguing that a standardized approach to care is critical across the different branches of the military. They also identify future Military and Veteran priorities that should guide current and future research efforts.

Wilde and colleagues provide an extensive review of neuroimaging findings across a range of modalities that have been used to examine TBI in Military and Veteran populations. This review emphasizes both the value (i.e., identifies a number of significant findings) and additional need (i.e., paucity of studies for some imaging modalities) for new studies in this area. The Cooper et al. manuscript is a timely review of the current TBI treatment literature summarize the Military and Veteran studies. This critical review provides positive preliminary evidence for rehabilitation. However, it is clear that this research is in its infancy and will benefit from applying clinical trial research designs that are aimed at understanding the specific features of rehabilitation that result in positive improvement of symptoms and cognitive function. Finally, in Bigler's review of the symptom validity research, a number of significant findings are noted and areas of future research are identified that could improve our ability to understand and utilize symptom validity measures. We included this article as it addresses an important, and often controversial, topic that could benefit from additional research, especially in mTBI. It is also clear that symptom validity in the Military and Veteran population will require careful consideration of a number of unique clinical features.

These reviews are followed by a number of original articles examining specific research questions related to Military/ Veteran TBI that have the potential to further advance our understanding of the challenges of working with these populations. The Jaramillo et al. paper provides unique insights into Iraq and Afghanistan Veterans with head injury utilizing advanced latent variable statistical analyses applied to population data sets from the Veteran Administration. This epidemiologic study of more than 300,000 Veterans clearly shows that TBI patients are often at risk for a number of significant comorbid conditions and that the combination of various comorbid conditions increase healthcare utilization. They also discuss the possibility of utilizing different or modified treatment approaches in patients across the different groups of patients as each group may have unique needs that can be more adequately addressed by the inclusion of specialities not frequently applied to TBI treatment. These results also emphasize the significant heterogeneity and complexity of this unique population of patients.

This epidemiology research is followed by three original manuscripts that address clinical questions regarding symptom presentation and personality profiles in Service Members and Veterans. In the first article, Lewis et al. examines the spatial distribution of lesion results from a large sample of Vietnam veterans to determine if prefrontal region injury results in worse anhedonia (a common symptom reported in TBI patients). Patients with lesions in the right ventrolateral regions of the brain appear to have additional symptom reporting that is consistent with anhedonia. The Miskey et al. and the Davenport et al. manuscripts both examine personality characteristics in Service Members and Veterans. These studies identify a number of personality features associated with clinical and imaging biomarkers commonly found in TBI and TBI associated comorbid conditions.

Using advanced diffusion imaging methods, Presson and colleagues explore the utility of diffusion spectrum imaging (DSI) combined with unique analyses in discriminating between TBI and control participants. This tract-based analysis represents an advancement in TBI diagnostics and provides impetus for future research. Diffusion tensor imaging (DTI) was also used in the Delano-Wood et al. manuscript to examine specific white matter tracts in the brain stem. Though there were no significant differences between the TBI and control samples, there was a difference in the functional significance of fractional anisotropy measures in the brain stem as related to various clinical variables of interest and post-concussive symptomatology. This study highlights an important feature of diffusion imaging metrics, namely the increased functional relevance that is often embedded in this complicated data and points to underlying neurobiological substrates of frequently reported symptoms in mTBI populations.

Utilizing functional imaging methods, the Schiebel et al. manuscript identifies unique patterns of activation that discriminate between TBI patients with clinically elevated posttraumatic stress symptoms and TBI patients with minimal or no clinical post-traumatic stress symptoms. In a study using positron emission tomography (PET), Raji and colleagues demonstrate functional differences between TBI and posttraumatic stress disorder (PTSD). Given the high comorbidity rates of PTSD in TBI and the significant overlap of presenting 
symptoms, these studies demonstrate a possible approach for distinguishing between these two disorders that could improve diagnosis and guide improved treatment strategies. The Sours et al. manuscript uses advanced resting state functional and spectroscopy imaging to examine the effects of TBI on the individual thalamic nuclei. This focused examination of the thalamus (already known to be affected in TBI) provides evidence for metabolic and functional imaging abnormalities in the acute (average 1 week post injury) period following injury. These abnormalities indicate metabolic and functional changes that might have implications for sensory and cognitive dysfunction often noted in TBI patients. McGlade and colleagues examine resting state functional imaging data for evidence of sex-based differences. Their data not only demonstrates a unique pattern of functional connectivity when comparing male and females service members, but the pattern in male participants was found to be related to behavioral measure of aggression. Combined, these studies provide growing evidence for the utility of using advanced imaging methods and statistical procedures to examine mTBI patients for underlying patterns of injury.

Imaging-related studies in military mTBI have dramatically increased over the past 5 to 10 years. This is not only attributable to the number of Service Members involved in the recent conflicts, but also to increased funding support for research in this area, improved access to military populations, technological advances in the field, and the influx of additional academic and clinical experts contributing to research in this area. The studies included in this special issue are a good illustration of improved methods and approaches to examining this patient population, and we note that there are several excellent projects currently underway that will provide additional important findings in the coming years. Some findings suggest that imaging may be useful in the identification of unique patterns of symptoms presentation, discrimination between patient groups, and plausible neurobiological insight into symptom presentation. This lends both credibility and objectivity to the study of mTBI and symptom presentation in Military and Veteran populations. In addition, these studies also provide preliminary evidence that $\mathrm{mTBI}$ and PTSD have different biological substrates despite seemingly overlapping symptom presentation. These types of findings are critical as they could guide the development of clinical interventions and treatments in this patient population. Importantly, these studies also highlight a number of limitations or gaps (i.e., lack of clinical trial treatment studies, longitudinal imaging studies, and consistent use of common data elements across studies; the need for improved access to normative data and the inability to examine the specific injury profile of individual patients) in the literature that will need to be addressed in order to provide better diagnosis, prognosis, and more complete treatment options to Service Members and Veterans who have served their country.

Compliance with ethical standards This article does not contain any studies with human participants performed by any of the authors.

Funding DFT and EAW are funded by grants from the DoD and VA (PT108802-SC106187 and 1W81XWH-13-2-0095). DFT also has additional funding from the MRMC (W81XWH-13-2-0025). EAW also has funding from the VA (1I01RX001062-01A1). SB is funded by the following grant awards: a VA MERIT award (1 I01 RX000928), a Department of Defense Office of the Congressionally Directed Medical Research Programs (CDMRP) award (X81XWH-07-CC-CSDoD), and a National Institutes of Health award (R01-NS078337).

Conflicts of interest DFT declares that he has no conflicts of interest. EAW declares that she has no conflicts of interest. SB declares that he has no conflicts of interests. SRM declares that he has no conflicts of interest.

\section{References}

Tate, D. F., Shenton, M. E., \& Bigler, E. D. (2012). Introduction to the brain imaging and behavior special issue on neuroimaging findings in mild traumatic brain injury. Brain Imaging and Behavior, 6(2), 103-107. 